\title{
FOLIA
}

Amazónica

Revista del Instituto de Investigaciones

de la Amazonía Peruana

\section{INFESTACIÓN DE Dolops discoidalis (CRUSTACEA: BRANCHIURA) EN Pseudoplatystoma punctifer (SILURIFORMES: PIMELODIDAE), Colossoma macropomum (CHARACIFORMES: SERRASALMIDAE) Y Brochis multiradiatus (SILURIFORMES: CALLICHTHYIDAE)}

\author{
Karla Leslie URIBE CHUQUIVAL ${ }^{1}$, Lucero Andrea ROMAINA CACHIQUE ${ }^{1}$, \\ César Augusto VARGAS DE PINA ${ }^{1}$, José Carlos ZUMAETA CACHIQUE ${ }^{2}$, \\ Germán Augusto MURRIETA MOREY*
}

1 Universidad Nacional de la Amazonía Peruana (UNAP), Sargento Lores, 385, Iquitos, Perú.

2 Instituto de Investigaciones de la Amazonía Peruana (IIAP), Laboratorio de Parasitología y Sanidad Acuícola, Carretera lquitos-Nauta Km 4,5, San Juan Bautista, 0784, Iquitos, Perú.

* Correo electrónico: germantiss1106@gmail.com

\section{RESUMEN}

El estudio se realizó en las instalaciones del Centro de Investigaciones Fernando Alcántara Bocanegra (CIFAB) del Instituto de Investigaciones de la Amazonía Peruana (IIAP), Iquitos; con el objetivo de conocer el comportamiento parasitario de Dolops discoidalis (Bouvier, 1899) (Crustacea: Branchiura) en tres especies de peces. El muestreo se realizó durante los meses de noviembre y diciembre del 2018. Para este estudio se extrajeron individuos de esta especie de parásitos de la superficie corporal de ejemplares adultos de Pseudoplatystoma punctifer (Castelnau, 1855), criados en cautiverio en las instalaciones del CIFAB. Los parásitos colectados fueron llevados al laboratorio de Parasitología y Sanidad Acuícola del IIAP para ejecutar dos experimentos relacionados al comportamiento parasitario de $D$. discoidalis en tres especies de peces: $P$. punctifer, Colossoma macropomum (Cuvier, 1816) y Brochis multiradiatus (Orcés V., 1960). El experimento I consistió en colocar un ejemplar de cada especie de pez en un acuario de vidrio (4 réplicas) y distribuir 18 ejemplares de $D$. discoidalis por unidad experimental con la finalidad de determinar el comportamiento parasitario de esta especie. Para el experimento II, se 
colocaron dos ejemplares de C. macropomum en dos acuarios diferentes y cuatro ejemplares de B. multiradiatus en un acuario, con 11 parásitos en cada una de las unidades experimentales. Los resultados del experimento I mostraron a todos los individuos de $D$. discoidalis parasitando únicamente a P. punctifer, revelando una marcada afinidad por esta especie de pez. En el experimento II se observó que el parásito $D$. discoidalis ante la ausencia de $P$. punctifer, es capaz de parasitar a ejemplares de C. macropomum y B. multiradiatus detectando entre uno a dos parásitos por pez parasitado, revelando muy baja afinidad. Se recomienda realizar estudios histológicos para determinar el grado de daño que causa este parasito en los peces, principalmente en P. punctifer, especie que mostró alta susceptibilidad ante $D$. discoidalis.

PALABRAS CLAVE: branchiuros, ectoparásitos, infestación, parásito de peces.

\title{
INFESTATION OF Dolops discoidalis (CRUSTACEA: BRANCHIURA) IN Pseudoplatystoma punctifer (SILURIFORMES: PIMELODIDAE), Colossoma macropomum (CHARACIFORMES: SERRASALMIDAE) AND Brochis multiradiatus (SILURIFORMES: CALLICHTHYIDAE)
}

\begin{abstract}
This study was carried out at the facilities of the "Centro de Investigaciones Fernando Alcántara Bocanegra (CIFAB) of the "Instituto de Investigaciones de la Amazonía Peruana (IIAP), Iquitos; with the objective of knowing the parasitic behavior of Dolops discoidalis (Bouvier, 1899) (Crustacea: Branchiura) in three fish species. The sampling was carried out during the months of November and December 2018. For this study, individuals of this parasitic species were extracted from the body surface of adult specimens of Pseudoplatystoma punctifer (Castelnau, 1855), bred in captivity at the CIFAB facilities. The collected parasites were taken to the laboratory of "Parasitología y Sanidad Acuícola" of the institute (IIAP) to perform two experiments evaluating the parasitic behavior of $D$. discoidalis in three fish species: P. punctifer, Colossoma macropomum (Cuvier, 1816) and Brochis multiradiatus (Orcés V., 1960). Experiment I consisted of placing one specimen of each species of fish in a glass aquarium (4 replicas) and distributing 18 specimens of $D$. discoidalis per experimental unit in order to determine the parasitic behavior of this species. For experiment II, 2 specimens of $C$. macropomum were placed in two different aquariums and 4 specimens of B. multiradiatus in one aquarium, with 11 parasites in each of the experimental units. The results of Experiment I showed
\end{abstract}


all individuals of $D$. discoidalis only parasitized $P$. punctifer, revealing a marked affinity for this species. In Experiment II it was observed that $D$. discoidalis, in the absence of $P$. punctifer, is capable of parasitizing C. macropomum and $B$. multiradiatus specimens, detecting between 1 to 2 parasites per parasitized fish, revealing very low affinity. Histological studies are recommended to determine the degree of damage caused by this parasite in fish, mainly in $P$. punctifer, a species that showed high susceptibility to $D$. discoidalis.

KEYWORDS: fish parasite, branchiurans, ectoparasites, infestation. 


\section{INTRODUCCIÓN}

El desarrollo de la actividad acuícola promueve el aumento de prácticas inadecuadas que desconocen el adecuado cuidado con el medio ambiente y la salud de los animales. Los sistemas intensivos a menudo se asocian con un aumento de la densidad y el volumen de la población y el uso masivo de alimentos artificiales, frecuentemente de mala calidad. Todas estas características, actúan directamente sobre la relación patógeno / ambiente / hospedero (García et al., 2013). Esta relación se da de forma equilibrada en el medio natural, a diferencia de los sistemas de crianza en cautiverio como: centros piscícolas, acuarios comerciales, donde pueden actuar invariablemente favoreciendo el desarrollo de patógenos, afectando las características ambientales y los mecanismos de defensa del hospedador (García et al., 2013).

Pseudoplatystoma punctifer (Castelnau, 1855) (Familia: Pimelodidae) se caracteriza por su amplia distribución en la cuenca del Amazonas y es considerada una de las especies comercialmente más importantes (Crepaldi et al., 2006). Alcanza más de $100 \mathrm{~cm}$ de longitud total, su carne tiene un alto valor comercial y es popular en los mercados amazónicos debido al sabor y ausencia de espinas intramusculares, mientras que ejemplares juveniles son muy apreciadas en el mercado ornamental (Núñez et al., 2011). En los últimos años, esta especie ha tomado interés en el cultivo intensivo, sumándose a otras especies que forman parte de la industria acuícola en América del Sur (Núñez, 2009), y fuera de su área de distribución original (Dabrowski et al., 2008).

Colossoma macropomum (Cuvier, 1816) (Characiformes: Serrasalmidae), conocida como gamitana, es una especie endémica de la cuenca del Amazonas, considerada como el segundo pez de mayor tamaño en América del Sur (AraujoLima \& Goulding, 1997). Esta especie puede alcanzar arriba de $90 \mathrm{~cm}$ de longitud y $30 \mathrm{Kg}$ de peso total, siendo altamente apreciada y demandada en los mercados de la Amazonía, y considerada como un pez de importancia en la acuicultura amazónica (Gomes et al., 2006).

La familia Callichthyidae alberga a numerosas especies con alta demanda y marcada apreciación en el mercado de exportación de peces ornamentales. En la Amazonía, son capturados en riachuelos, ríos y lagos (Sánchez et al., 2011). Dentro de este grupo de peces, Brochis multiradiatus (Orcés V. 1960), conocida popularmente como "coridora jumbo" es muy requerida por exportadores de peces ornamentales, constituyendo un recurso íctico de exportación a diferentes países del mundo (Sánchez et al., 2011).

Las infecciones parasitarias son una de las principales causas de pérdidas económicas en centros piscícolas (Tavares-Dias \& Martins, 2017). Son de gran relevancia en los trópicos debido a la influencia del cambio climático, facilitando su propagación (Malta, 1983; Malta, 1984). Entre los parásitos, los branchiuros son de particular interés y pueden ser difíciles de manejar debido a su comportamiento típico de moverse de un lugar a otro para alimentarse y poner huevos (Malta, 1983; Malta, 1984). En estos parásitos, el aparato oral tiene ganchos, estilete y espinas para la fijación y alimentación. El estilete perfora la piel del huésped, succionando sangre y células epiteliales. El hábito hematófago de estos parásitos causa anemia en los peces, mientras que la histofagia causa la inflamación del tegumento cutáneo, lo que permite el establecimiento de infecciones secundarias (Malta, 1984; Malta \& Varella, 2000).

Teniendo en cuenta la importancia de las especies de peces mencionadas para la acuicultura y los riesgos de infestación por parte de los branchiuros, el presente estudio tuvo como objetivos conocer el nivel de infestación a 
través del cálculo de los índices parasitarios de $D$. discoidalis, y el comportamiento parasitario ante sus respectivos hospederos.

\section{MATERIAL Y MÉTODOS}

Para iniciar los experimentos, previamente fue necesario colectar ejemplares de $D$. discoidalis. Para ello, se realizó un muestreo en un estanque piscícola ubicado en las instalaciones del Centro de Investigaciones Fernando Alcántara Bocanegra del Instituto de Investigaciones de la Amazonía Peruana (IIAP), Iquitos, Perú, entre las coordenadas geográficas $3^{\circ} 49^{\prime} 02.35^{\prime \prime} \mathrm{S} /$ $73^{\circ} 19^{\prime} 14.43^{\prime \prime}$ W. Fueron utilizados 27 ejemplares adultos de $P$. punctifer, de los cuales se colectaron un total de 89 branchiuros. Los parásitos fueron conservados vivos en un envase de plástico con agua para su posterior uso en los experimentos de laboratorio.

Adicionalmente, se calcularon los índices parasitarios de los 27 ejemplares de P. punctifer de acuerdo a Bush et al. (1997): prevalencia (P), Intensidad (I); Intensidad media de infestación (IMI) y la Abundancia media de infestación (AM).

Los experimentos se llevaron a cabo en el Laboratorio de Parasitología y Sanidad Acuícola del IIAP. En los experimentos de laboratorio se emplearon cuatro ejemplares de $P$. punctifer con 20,38 $\pm 0,50 \mathrm{~cm}$. de longitud estándar y $38,30 \pm 3,12$ g. de peso; cuatro ejemplares de $C$. macropomum con $16,88 \pm 0,43 \mathrm{~cm}$. de longitud estándar y $71,39 \pm 5,17$ g. de peso y cuatro ejemplares de $B$. multiradiatus con 8,68 $\pm 0,56$ cm. de longitud estándar y 18,18 $\pm 13,52$ g. de peso (Figura 1).
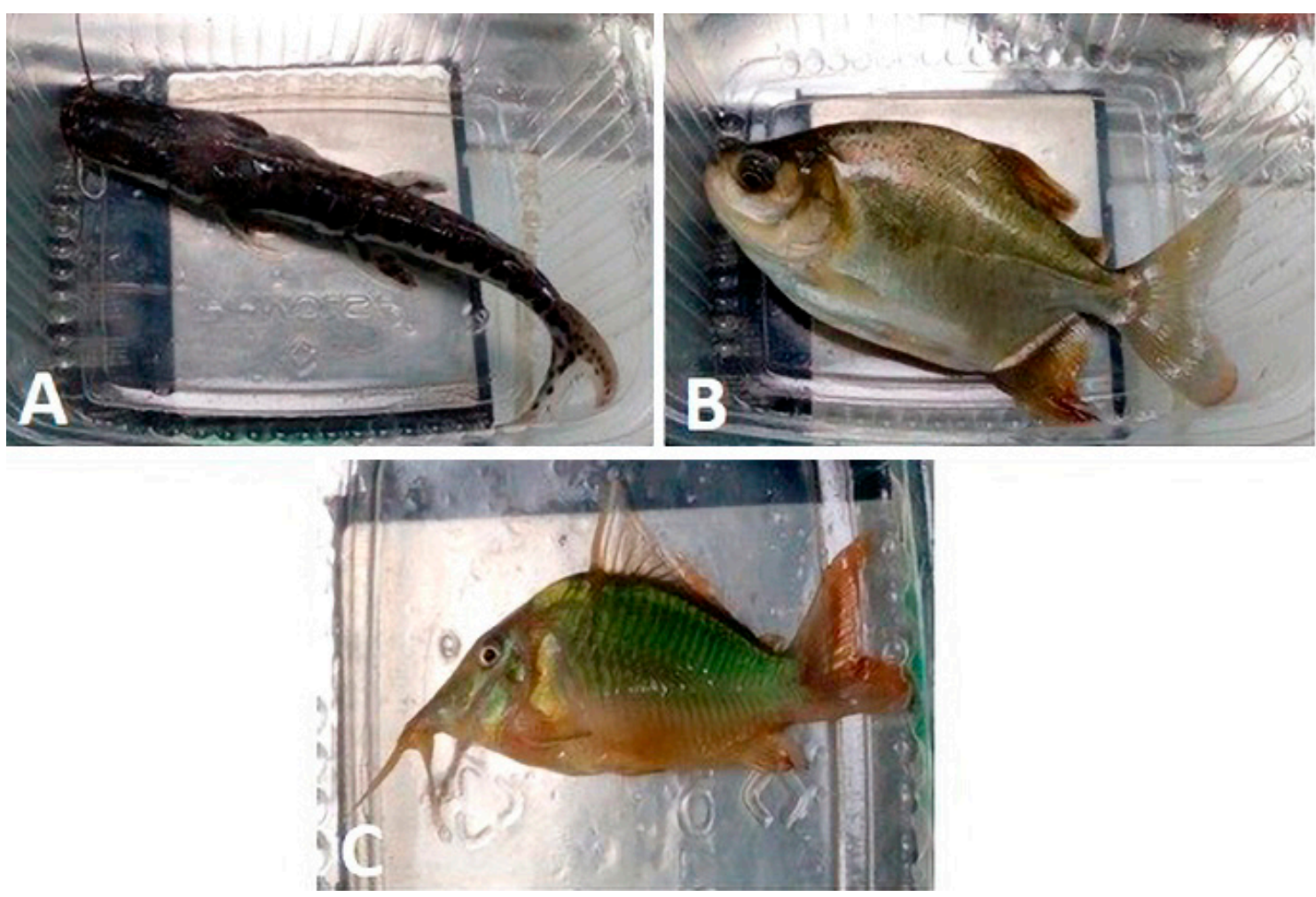

Figura 1: A. Pseudoplatystoma punctifer (Castelnau, 1855) "Doncella", B. Colossoma macropomum (Cuvier, 1816) "Gamitana" y C. Brochis multiradiatus (Orcés V., 1960) "Coridora jumbo". 


\section{Experimento I}
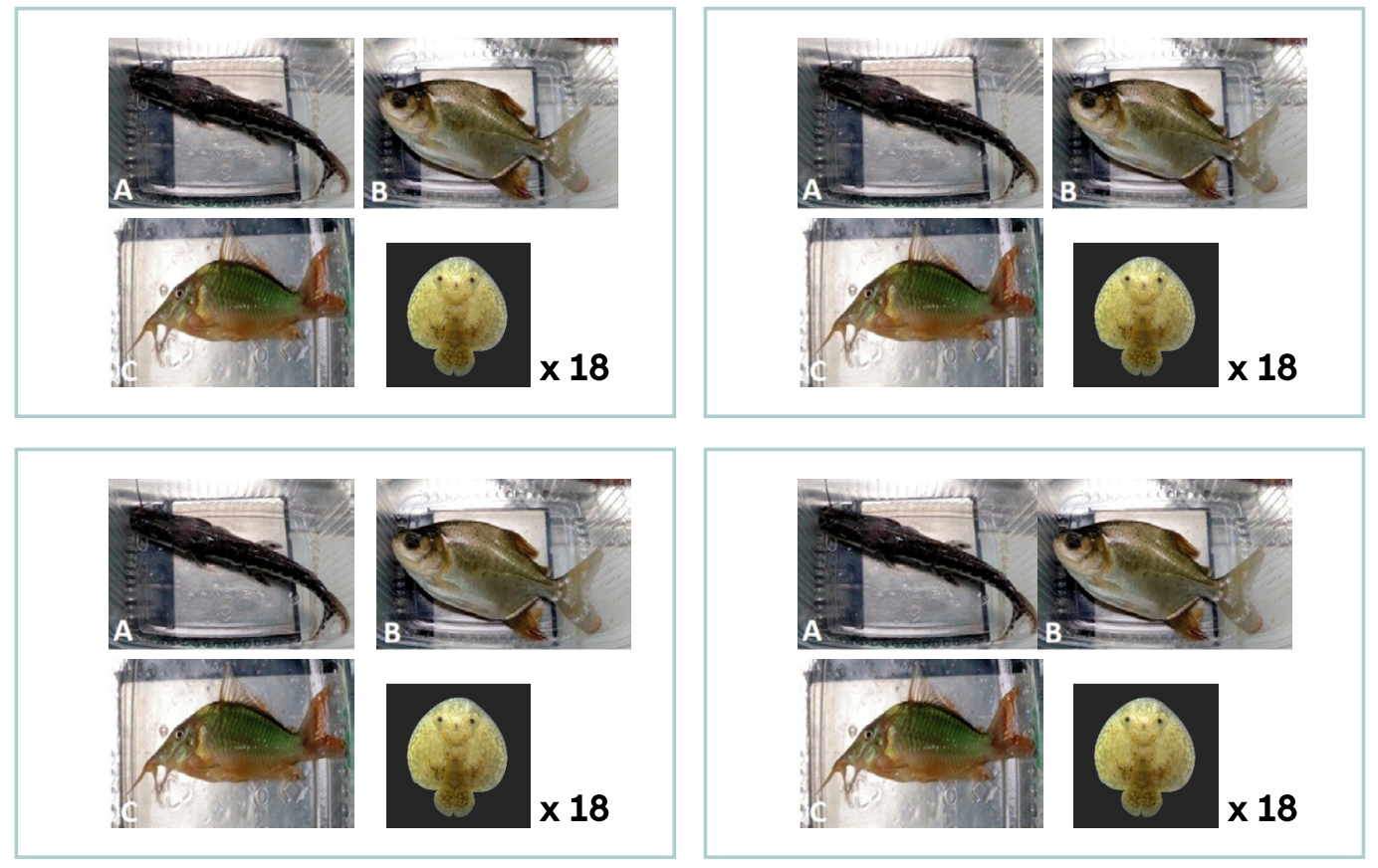

\section{Experimento II}
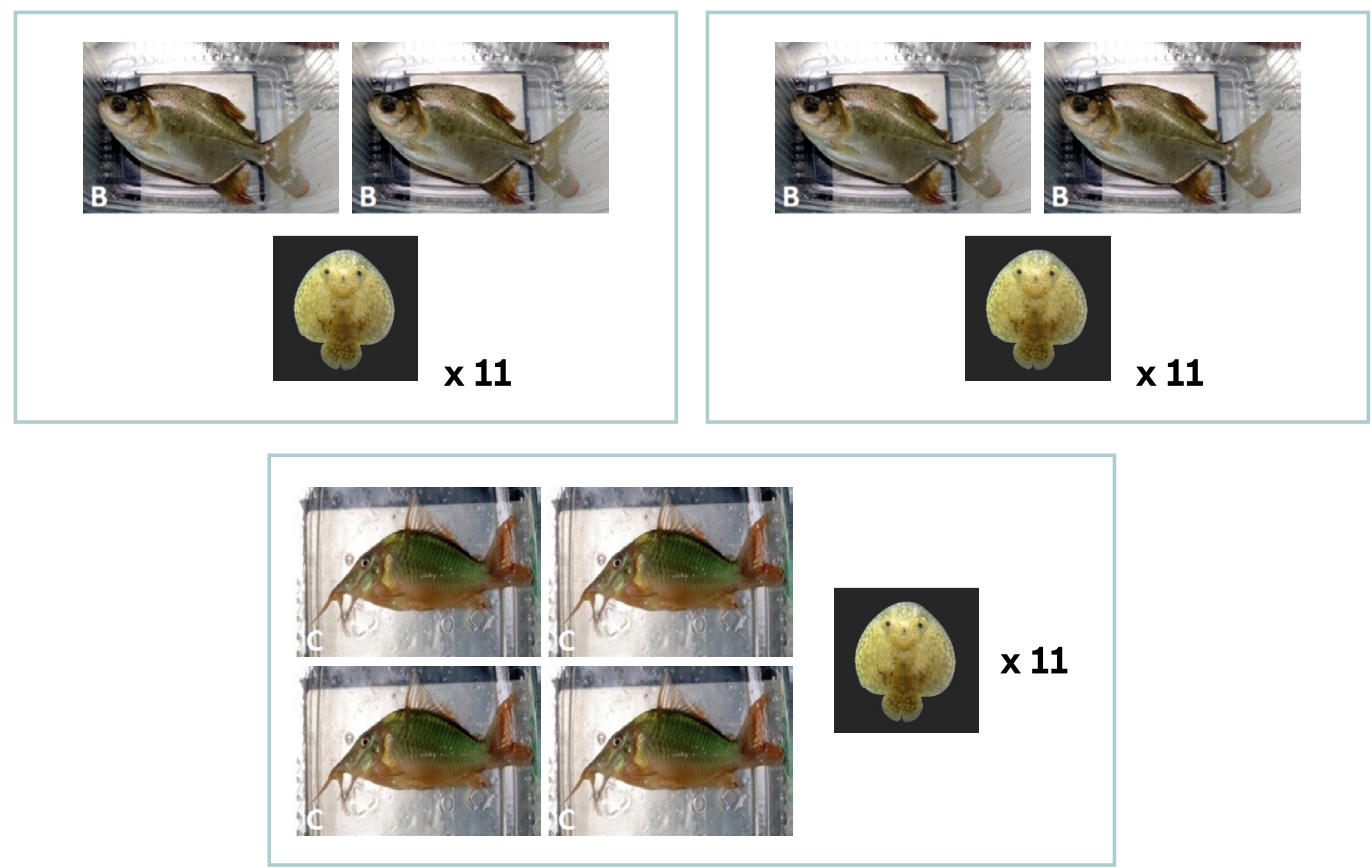

Figura 2. Esquema de los experimentos realizados. Experimento I. Unidad experimental con un ejemplar de Pseudoplatystoma punctifer (Castelnau, 1855), Colossoma macropomum (Cuvier, 1816) y Brochis multiradiatus (Orcés V., 1960) conjuntamente con 18 ejemplares de Dolops discoidalis (Bouvier, 1899). Experimento II. Unidad experimental con dos ejemplares de Colossoma macropomum y 11 ejemplares de Dolops discoidalis, unidad experimental con cuatro ejemplares de Brochis multiradiatus conjuntamente con 11 ejemplares de

Dolops discoidalis. 
Los experimentos son descritos a continuación:

Experimento I: Se emplearon cuatro acuarios (unidades experimentales) de 40x30×30 cm, cada uno con $20 \mathrm{~L}$ de agua donde se colocó un ejemplar de cada especie de pez (P. punctifer, C. macropomum, B. multiradiatus) por unidad experimental. En cada unidad experimental se colocaron 18 ejemplares de $D$. discoidalis, haciendo un total de 72 parásitos. El comportamiento parasitario fue observado durante ocho días (Figura 2).

Experimento II: Para este experimento se emplearon los mismos peces del Experimento I, a excepción de los cuatro ejemplares juveniles de $P$. punctifer. Es decir, se utilizaron cuatro ejemplares de $C$. macropomum distribuidos en dos acuarios a razón de dos peces y cuatro ejemplares de $B$. multiradiatus distribuidos todos en un mismo acuario. En cada unidad experimental se colocaron 11 parásitos, haciendo un total de 33 parásitos. El comportamiento parasitario fue observado durante cinco días (Figura 2).

Los valores de los parámetros físicos químicos del agua como la temperatura $\left({ }^{\circ} \mathrm{C}\right)$, la concentración de oxígeno disuelto (mg/L), la conductividad $(\mu \mathrm{S} / \mathrm{cm})$ y el $\mathrm{pH}$, se registraron diariamente desde el inicio hasta el final de ambos experimentos, siendo estos en horas de la mañana, para ello se utilizó equipos como el Oxímetro marca YSI modelo 550A; y el Multiparámetro marca HACH modelo HQ400D
Multi. Además, los acuarios fueron forradas con cartulina negra para evitar el estrés de los peces ante la presencia humana. Cada acuario fue implementado con bombas de aire, los cuales funcionaban las 24 horas para mantener un flujo constante de oxígeno.

\section{RESULTADOS}

\section{DESCRIPCIÓN MORFOLÓGICA DE DOLOPS}

\section{DISCOIDALIS}

El branquiuro Dolops discoidalis se registró parasitando la superficie dorsal y ventral del cuerpo de los peces (Figura 3). Las principales características de esta especie son: caparazón orbicular en forma más ancha que larga y más estrecha anteriormente. Los lóbulos posteriores del caparazón están redondeados, llegando un poco más allá de la base del abdomen; los lóbulos no cubren el tórax, dejándolo visible en toda su extensión. El caparazón cubre los cuatro pares de patas en casi todos los especímenes. Existe dimorfismo sexual, caracterizándose ejemplares machos por la presencia de testículos trilobados, siendo el lóbulo externo más pequeño que los otros (Figura 4A). Ejemplares hembras presentan espermatecas visibles (Figura 4B). Individuos juveniles son pequeños, sin órganos sexuales bien definidos. Las medidas morfométricas de hembras, machos y juveniles de ejemplares de $D$. discoidalis se muestran en la Tabla 1.

Tabla 1. Medidas morfométricas de Dolops discoidalis según sexo. $q=$ hembras, $\lambda=$ machos, Juv $=$ juveniles.

\begin{tabular}{ccccccc}
\hline & \multirow{5}{c}{ Promedio \pm DS } \\
\cline { 3 - 7 } & $\begin{array}{c}\text { Sexo } \\
\text { - }+ \text { - Juv }\end{array}$ & $\begin{array}{c}\text { Longitud Total } \\
(\mathrm{LT})\end{array}$ & $\begin{array}{c}\text { Longitud de } \\
\text { Caparazón (LC) }\end{array}$ & $\begin{array}{c}\text { Ancho de } \\
\text { Caparazón (AC) }\end{array}$ & $\begin{array}{c}\text { Ancho de } \\
\text { Abdomen (AAb) }\end{array}$ & $\begin{array}{c}\text { Longitud de } \\
\text { Abdomen (LAb) }\end{array}$ \\
\hline 1 & + & $11,24 \pm 1,92$ & $8,50 \pm 1,49$ & $9,75 \pm 1,78$ & $4,08 \pm 0,88$ & $3,06 \pm 0,74$ \\
2 & 0 & $12,89 \pm 1,83$ & $10,64 \pm 1,50$ & $11,57 \pm 1,73$ & $3,77 \pm 0,57$ & $2,79 \pm 0,65$ \\
3 & Juv & $2,33 \pm 2,17$ & $1,65 \pm 1,53$ & $1,94 \pm 1,81$ & $0,46 \pm 0,51$ & $0,25 \pm 0,34$ \\
\hline
\end{tabular}



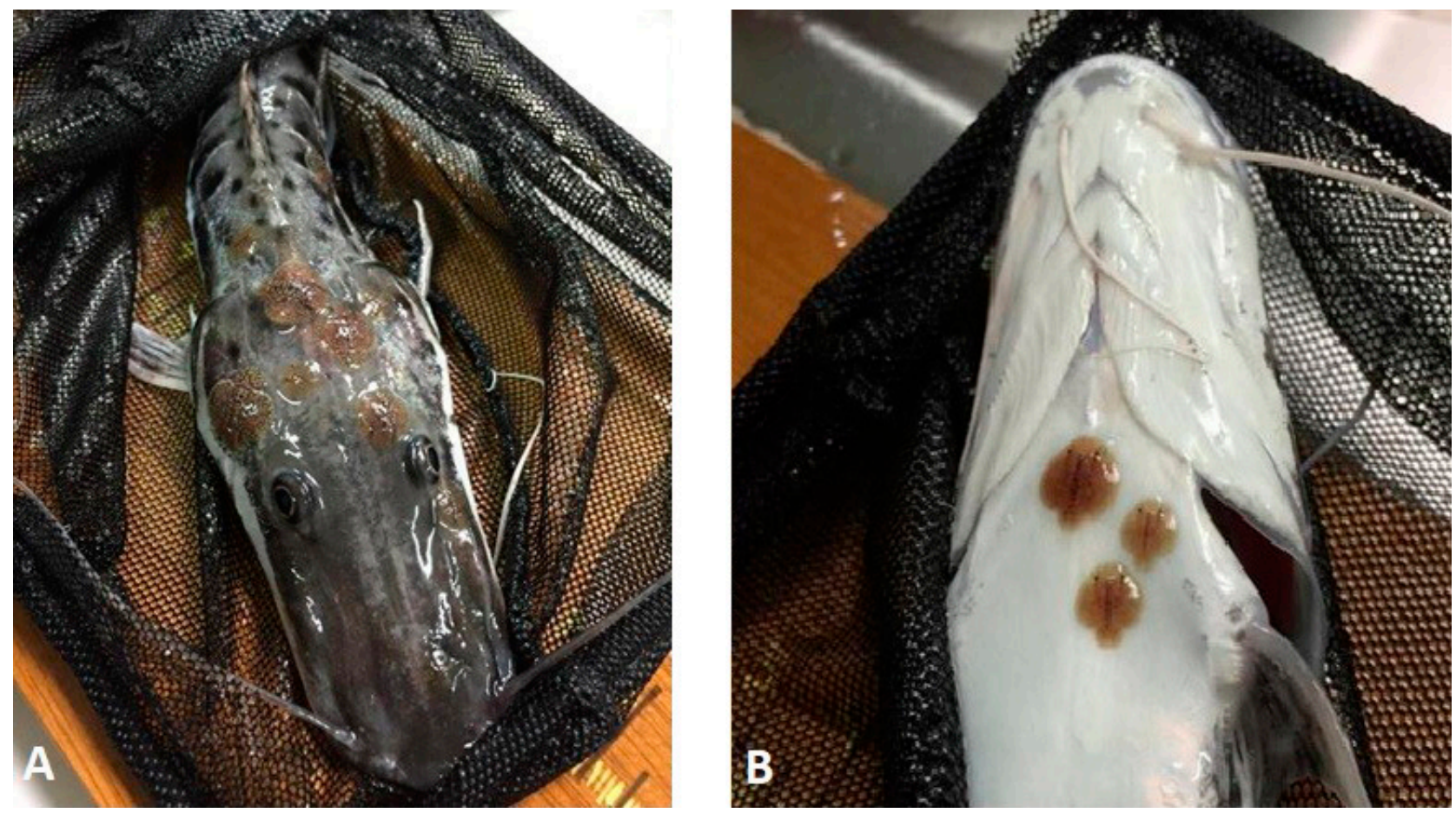

Figura 3. Pseudoplatystoma punctifer (Castelnau, 1855), "Doncella" parasitados por Dolops discoidalis (Bouvier, 1899), en parte dorsal (A) y en la parte ventral (B).
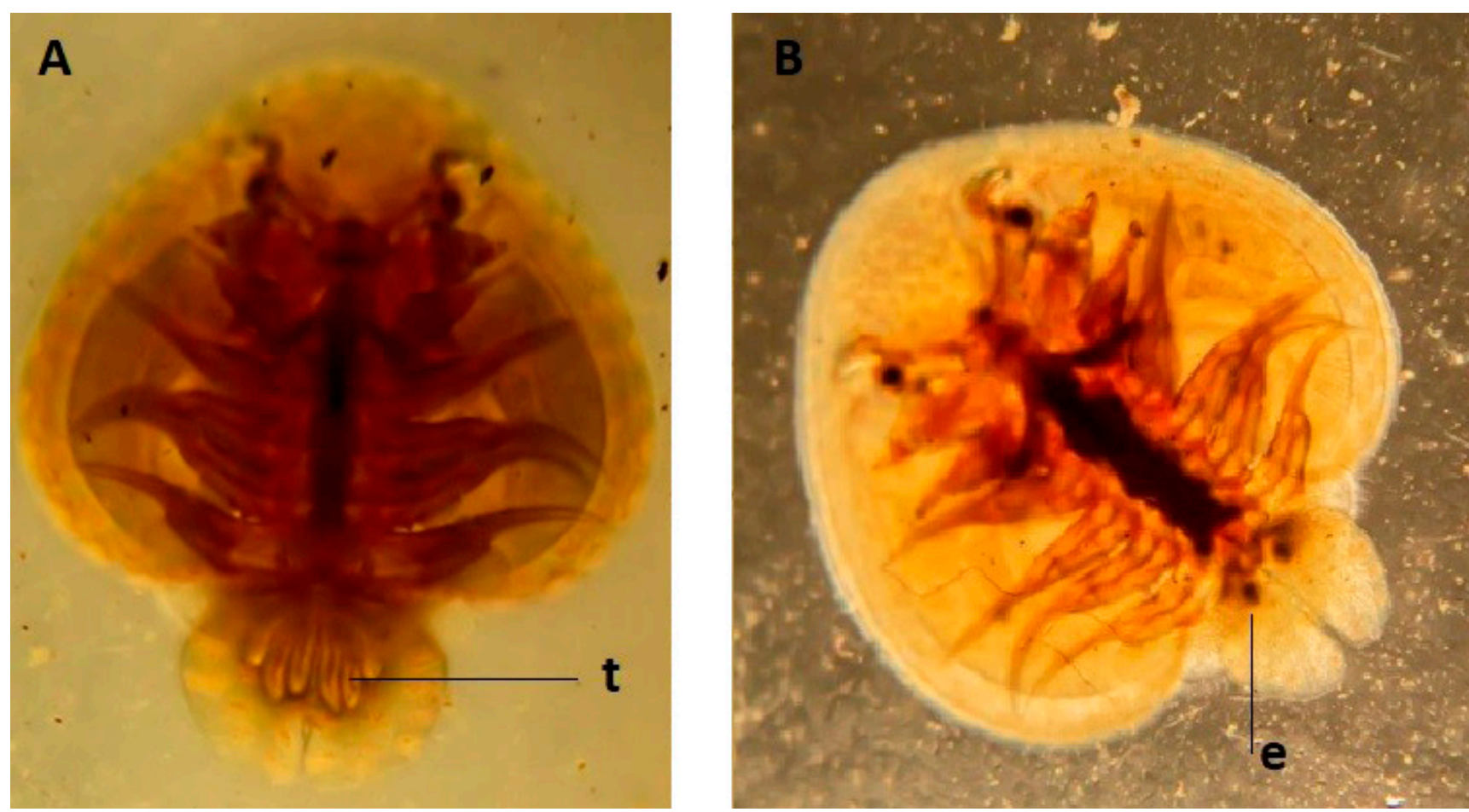

Figura 4. A. Ejemplar macho de Dolops discoidalis (Bouvier, 1899) evidenciado los testículos (t). B. Ejemplar hembra de $D$. discoidalis evidenciando las espermatecas (e). 

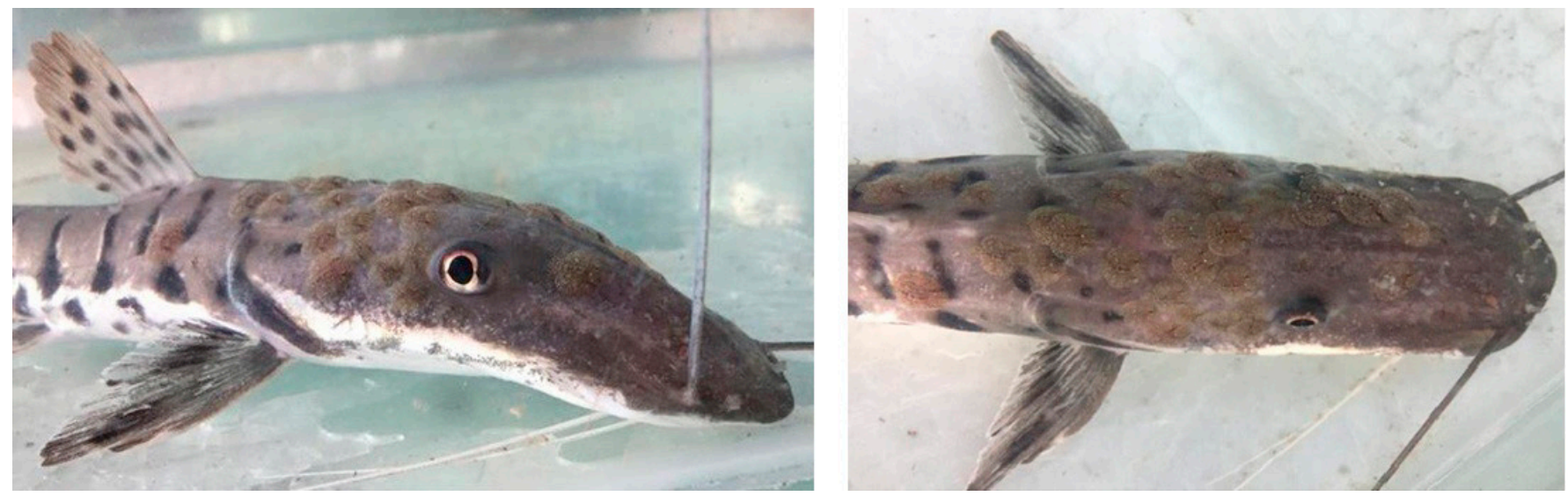

Figura 5. Pseudoplatystoma punctifer (Castelnau, 1855) “Doncella” parasitadas por numerosos especímenes de Dolops discoidalis (Bouvier, 1899), en el Experimento I.
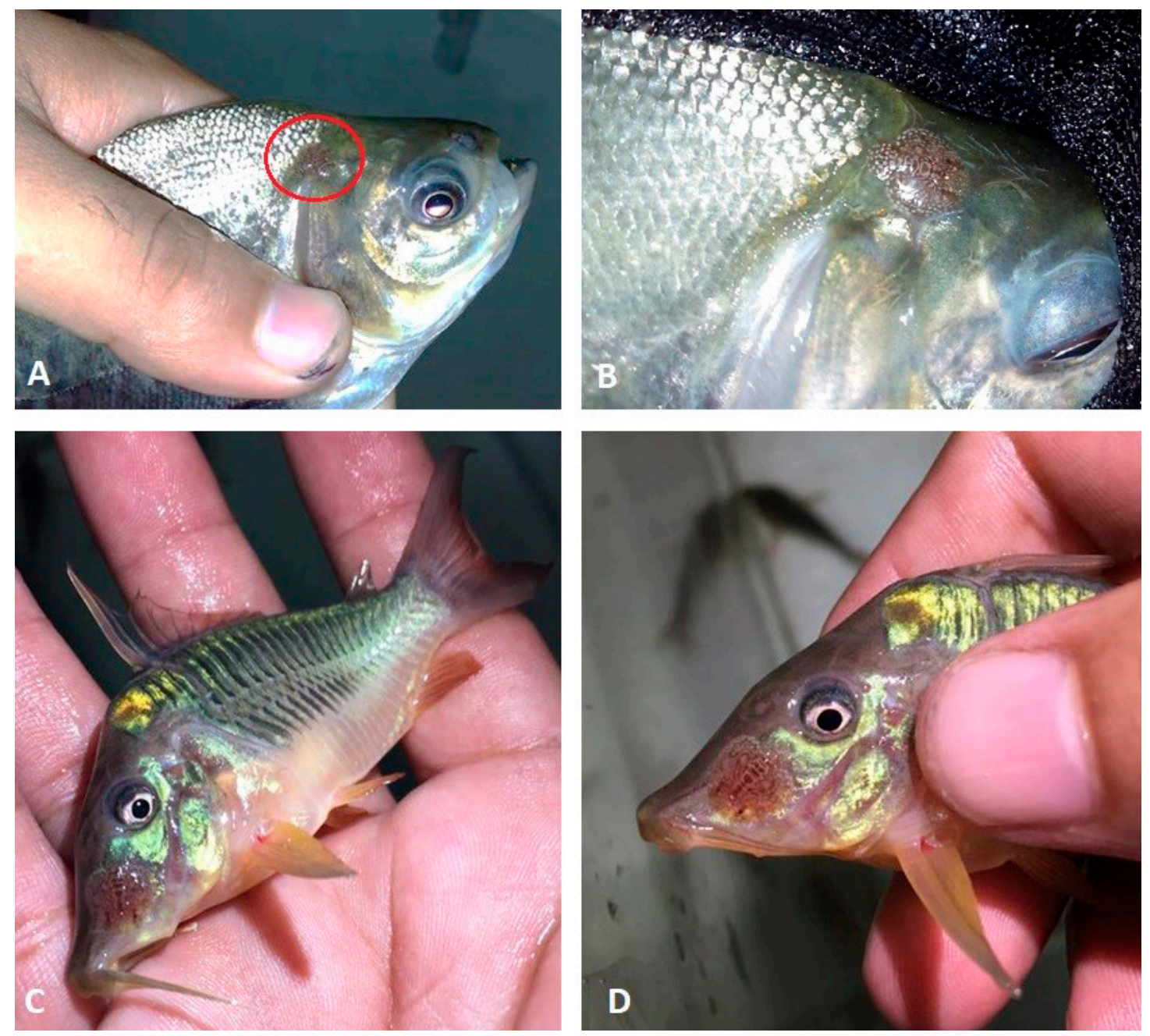

Figura 6. A y B. Dolops discoidalis (Bouvier, 1899) parasitando Colossoma macropomum (Cuvier, 1816), C y D. Dolops discoidalis parasitando Brochis multiradiatus (Orcés V., 1960). 


\section{CÁLCULO DE LOS ÍNDICES PARASITARIOS}

Del total de los 27 ejemplares de P. punctifer, solo 18 peces fueron parasitados, los cuales registraron una prevalencia del 66,67 \% (Tabla 2).

Tabla 2. Índices parasitarios de Dolops discoidales colectados de ejemplares de Pseudoplatystoma punctifer procedentes de estanque de cultivo del Centro de Investigaciones Fernando Alcántara Bocanegra, IIAP.

\begin{tabular}{lc}
\hline ÍNDICES PARASITÁRIOS & VALORES \\
\hline Prevalencia (\%) & 66,67 \\
Intensidad (I) & $89(1-9)$ \\
Intensidad Media (IM) & 4,94 \\
Abundancia Media (AM) & 3,30 \\
\hline
\end{tabular}

De los 89 especímenes de $D$. discoidalis que se recolectaron en el muestreo del estanque del Centro de Investigaciones Fernando Alcántara Bocanegra, IIAP, se pudo determinar que 21 eran machos, 22 hembras y 46 inmaduros.

\section{COMPORTAMIENTODEDOLOPSDISCOIDALIS} EN TRES ESPECIES DE PECES

Experimento I. Durante los ocho días que duró el Experimento I se observó que los parásitos $D$. discoidalis parasitaron únicamente a $P$. punctifer. Estos parásitos al desplazarse de su lugar de fijación dejaban una marca de sangre coagulada en la piel del pez (Figura 5).

Experimento II. Durante los cinco días del experimento I se observó que el parásito $D$. discoidalis ante la ausencia del P. punctifer, es capaz de parasitar otras especies de peces, sin embargo, este parasito no tiene afinidad marcada en hospedar a los otros peces ya que sólo se registraron entre 1 o 2 parásitos en la superficie corporal de los ejemplares de C. macropomum y B. multiradiatus (Figura 6). Además se observó que los parásitos se adhirieron a las paredes del acuario, observando también la muerte de algunos individuos, atribuyéndose a la ausencia de su hospedero habitual (P. punctifer).

\section{VALORES DE LOS PARÁMETROS FÍSICOS Y QUÍMICOS}

Los valores promedios de los parámetros físicos y químicos del agua durante el desarrollo de ambos experimentos no mostraron amplia variación manteniéndose en los valores normales para el cultivo de peces amazónicos, presentando ligeras variaciones que no afectaron aparentemente en el desarrollo de las investigaciones (Tabla 3).

Tabla 3. Registro de los de los Parámetros Físicos Químicos del agua según el Experimento I y |l.

\begin{tabular}{lcccc}
\hline \multirow{2}{*}{$\begin{array}{c}\text { Parámetros Físicos } \\
\text { Químicos }\end{array}$} & \multicolumn{2}{c}{ Experimento I } & \multicolumn{2}{c}{ Experimento II } \\
\cline { 2 - 5 } & Promedio \pm DS & $\begin{array}{c}\text { Variación } \\
\text { (Mín. - Máx.) }\end{array}$ & Promedio \pm DS & $\begin{array}{c}\text { Variación } \\
\text { (Mín. - Máx.) }\end{array}$ \\
\hline Temperatura $\left({ }^{\circ} \mathrm{C}\right)$ & $28,0 \pm 0,4$ & $27,1-28,5$ & $26,7 \pm 0,2$ & $26,5-27,0$ \\
Oxígeno $(\mathrm{mg} / \mathrm{L})$ & $3,65 \pm 0,8$ & $2,34-4,79$ & $4,69 \pm 0,9$ & $3,32-5,84$ \\
Conductividad $(\mu \mathrm{S} / \mathrm{cm})$ & $45,5 \pm 2,2$ & $41,2-50,7$ & $39,8 \pm 5,6$ & $31,9-49,1$ \\
$\mathrm{pH}$ & $6,14 \pm 0,4$ & $5,25-6,76$ & $6,36 \pm 0,2$ & $6,14-6,86$ \\
\hline
\end{tabular}




\section{DISCUSIÓN}

La literatura revisada considera que Dolops discoidalis parasita a pimelódidos: Leiarius marmoratus (Gill, 1870), Phractocephalus hemiliopterus (Bloch \& Schneider, 1801), Pseudoplatystoma fasciatum (Linnaeus, 1766), como se describe en el estudio de crustáceos que parasitan peces de Brasil (Luque et al., 2013). En el presente estudio, $D$. discoidalis fue registrado parasitando a especímenes de $P$. punctifer cultivados en un estanque piscícola. De acuerdo a los experimentos de comportamiento realizados, ejemplares de $D$. discoidalis tuvieron marcada afinidad por los individuos de P. punctifer; ya que cuando fueron sometidos a la infestación en las unidades experimentales, parasitaron únicamente a estos peces. La afinidad de este parásito por esta especie de pez fue reforzada en el segundo experimento, donde los parásitos ubicados en las unidades experimentales con ejemplares de $C$. macropomum y B. multiradiatus no mostraron interés por estos peces, obviando el parasitismo ante la ausencia de $P$. punctifer. De esta forma, se corrobora la afinidad de este parásito por hospederos de la familia Pimelodidae.

Entre los parásitos, la especificidad se considera un fenómeno dinámico, donde los huéspedes y los parásitos se adaptan unos a otros. Algunas especies de parásitos presentan alto grado de especificidad parasitaria siendo restringidas a una única especie de hospedero, mientras que otras presentan baja especificidad, ocurriendo en varias especies de hospederos (Malta, 1982). El parásito D. discoidalis presenta una especificidad parasitaria muy baja, pues durante el período de colectas, se encontró parasitando ocho especies de peces incluidos en cuatro familias: cinco especies de la familia Pimelodidae, una de la Erythinidae, una de la Cichlidae y una de Osteoglossidae (Malta 1982).
En el presente estudio, los resultados difieren de Malta (1982), ya que los ejemplares de $D$. discoidalis mostraron marcada preferencia por individuos de P. punctifer, no parasitando a $C$. macropomum (Serrasalmidae) y B. multiradiatus (Callichthyidae). En tal sentido, se asume que $D$. discoidalis es específico de P. punctifer.

Dolops discoidalis tiene una preferencia por "peces lisos" (peces de cuero ausentes de placas óseas o escamas), pues cinco de las ocho especies que ocurrieron como sus hospederos fueron "peces lisos", sólo tres con escamas (Malta, 1982). Los resultados del presente trabajo, se adecuan al enunciado del autor (Malta, 1982). De esta forma, nuestros datos coinciden con lo previamente mencionado con respecto a la preferencia de $D$. discoidalis por "peces lisos".

Las infestaciones de branchiuros son un importante desafío para los piscicultores. Incluso una leve infestación por estos parásitos es perjudicial en peces de cultivo debido a las densidades de siembra durante la crianza, lo que facilita la rápida propagación de parásitos y puede ocurrir un brote severo. Además, las lesiones en la piel causadas por estos parásitos, comprometen el estado de salud de los peces y pueden llevar a reducir la respuesta inmunológica frente a otras enfermedades (Tavares-Dias et al., 2007).

En un estudio realizado por Morey \& Satalaya (2019) se reportó al branchiuro D. discoidalis parasitando a ejemplares de $P$. punctifer criados en cautiverio. Aunque los índices parasitarios reportados fueron bajos y no representaron la manifestación de alguna enfermedad o daño considerable en lospeces, se alerta tomar cuidados necesarios para evitar la proliferación masiva de los parásitos y consecuente manifestación de problemas sanitarios.

En un estudio realizado en un estanque de peces en Iquitos, Perú, se reportó una alta infestación de Argulus pestifer Ringuelet, 1948 en Brachyplatystoma tigrinum (Britski, 1981), donde 
las cargas de parásitos alcanzaron entre 12 y 30 peces parasitados (Alcántara et al., 2008). En ese estudio, se observó que los peces infestados tenían: erosiones en la piel, con pérdida de moco y tejido epitelial, hasta la exposición del hueso de la región cefálica, pérdida de masa corporal, reducción del factor de condición relativa y la muerte de algunos ejemplares. En los resultados del presente estudio, la carga de $D$. discoidalis sobre P. punctifer, C. macropomum y B. multiradiatus de acuerdo a los experimentos realizados fue baja. Los niveles observados no causaron daño mecánico a la piel ni estado nutricional y condición del pez. Sin embargo, la alta prevalencia de parásitos es causa de grave preocupación, ya que la posibilidad de una epizootia, en condiciones inadecuadas o cambios drásticos en los parámetros físicos y químicos del agua, pone en riesgo la producción de peces.

\section{REFERENCIAS BIBLIOGRÁFICAS}

Alcántara, F.; Chu-Koo, F.; Rodríguez, L.; Chávez, C.; Bernuy, A.; Barbarán, T.; Nuñez, J. 2008. Primer reporte de parasitismo de Brachyplatystoma tigrinum por Argulus pestifer en acuicultura. Folia Amazónica, 17: 99-102. DOI: https://doi. org/10.24841/fa.v17i1-2.272

Araujo-Lima, C.R.M.; Goulding, M. 1997. So fruitful fish: ecology, conservation, and aquaculture of the Amazon's tambaqui. Columbia University Press, New York. 157pp.

Bouvier, M.E.L. 1899. Les crustacés parasites du genre Dolops (Audouin). Bulletin de la Société philomathique de Paris, 10: 53-81.

Bush A.O.; Lafferty, K.D.; Lotz, J.M.; Shostak, A.W. 1997. Parasitology meets ecology on its own terms: Margolis et al. revisited. Journal of Parasitology, 83(4): 575-583.
Castelnau, F.L. 1855. "Poissons". In: Animaux Nouveaux or Rares Recueillis Pendant l'Expédition dans les Parties Centrales de l'Amérique du Sud de Rio de Janeiro a Lima, et de Lima au Para; Exécutée par ordre du Gouvernment Français Pendant les Années 1843 a 1847, Part 7, Zoology 2: 1-112.

Crepaldi, D.V.; Faria, P.M.C.; Teixeira, E.A.; Ribeiro, L.P. 2006. 0 surubim na aquacultura do Brasil. Revista Brasileira de Reprodução Animal, 30: 150-158.

Dabrowski, K.; Arslan, M.; Rinchard, J.; Palacios, E.M. 2008. Growth, maturation, induced spawning, and production of the first generation of South American catfish, Pseudoplatystoma sp., in North America. Journal of the World Aquaculture Society, 39: 174-183. DOI: https://doi.org/10.1111/ j.1749-7345.2008.00147.x

García, F.; Romera, D.M.; Gozi, K.S.; Onaka, E.M.; Fonseca, F.S.; Schalch, S.H.; Candeira, P.G.; Guerra, L.O.M.; Carmo, F.J.; Carneiro, D.J.; Martins, M.I.E.G.; Portella, M.C. 2013. Stocking density of Nile tilapia in cages placed in a hydroelectric reservoir. Aquaculture, 410411: 51-56. DOI: https://doi.org/10.1016/j. aquaculture.2013.06.010

Gomes, L.C.; Chagas, E.C.; Martins, H.; Roubach, R.; Ono, E.A.; Lourenço, J.N.P. 2006. Cage cultured of tambaqui (Colossoma macropomum) in a central Amazon floodplain lake. Aquaculture, 253: 374-384. DOI: https://doi.org/10.1016/j. aquaculture.2005.08.020

Luque, J.L.; Vieira, F.M.; Takemoto, R.M.; Pavanelli, G.C.; Eiras, J.C. 2013. Checklist of Crustacea parasitizing fishes from Brazil. Check List, 9(6): 1449-1470. DOI: https://doi. org/10.15560/9.6.1449

Malta, J.C.O. 1982. Os argulídeos (Crustacea: Branchiura) da Amazônia Brasileira. Aspectos 
da ecologia de Dolops discoidalis Bouvier, 1399 e Dolops bidentata Bouvier, 1899 (1). Acta Amazônica, 12(3): 521-528.

Malta, J.C.O. 1983. Os argulíddeos (Crustacea: Branchiura) da Amazônia brasileira 3. Aspectos da ecologia de Dolops striata Bouvier, 1899 e Dolops carvalhoi Castro, 1949. Acta Amazônica, 13(2): 299-306.

Malta, J.C.O. 1984. Os peixes de um lago de várzea da Amazônia Central (Lago Janauacá, Rio Solimões) e suas relações com os crustáceos ectoparasitas (Branchiura: Argulidae). Acta Amazônica, 14(4): 355-372.

Malta, J.C.O.; Varella, A.M.B. 2000. Argulus chicomendesi sp. n. (Crustacea: Argulidae) parasita de peixes da Amazônia brasileira. Acta Amazônica, 30(1): 481-489.

Morey, G.A.M.; Satalaya, A.H. 2019. Infestation of Dolops discoidalis Bouvier, 1899 (Branchiura:
Argulidae) on Pseudoplatystoma punctifer (Castelnau, 1855) (Siluriformes: Pimelodidae) from a fish pond in the Peruvian Amazon. Aquaculture, 500: 414-416. DOI: https://doi. org/10.1016/j.aquaculture.2018.10.038

Sánchez, H.; García, A.; Vásquez, J.; Alcántara, F. 2011. Peces ornamentales amazónicos. Iquitos. Instituto de Investigaciones de la Amazonía Peruana, 76 pp.

Tavares-Dias, M.; De Moraes, F.R.; Onaka, E.M.; Rezende, P.C.B. 2007. Changes in blood parameters of hybrid tambacu fsh parasitized by Dolops carvalhoi (Crustacea, Branchiura), a fish louse. Veterinarski Arhiv, 77(4): 355-363.

Tavares-Dias, M.; Martins, M.L. 2017. An overall estimation of losses caused by diseases in the Brazilian fish farms. Journal of Parasitic Diseases, 41: 913-918. DOI: https://doi. org/10.1007/s12639-017-0938-y

Recibido: 11 de agosto de 2020 Aceptado para publicación: 30 de setiembre de 2020

Esta obra está bajo una Licencia Creative Commons Atribución-NoComercial-SinDerivar 4.0 Internacional. 